\title{
Non-Hermitian Cavity Quantum Electrodynamics - Configuration Interaction Singles Approach for Polaritonic Structure with ab initio Molecular Hamiltonians
}

\author{
Jonathan McTague ${ }^{1}$ and Jonathan J. Foley IV ${ }^{1}$ \\ William Paterson University, Department of Chemistry, 300 Pompton Road, Wayne NJ 07470, \\ USA \\ (*Electronic mail: foleyj10@wpunj.edu)
}

(Dated: 20 August 2021)

We combine $a b$ initio molecular electronic Hamiltonians with a cavity quantum electrodynamics model for dissipative photonic modes and apply mean-field theories to the ground- and excited-states of resulting polaritonic systems. In particular, we develop a restricted Hartree-Fock theory for the mean-field ground-state and a non-Hermitian configuration interaction singles theory for mean-field excited-states of the molecular system strongly interacting with a photonic mode, and apply these methods to several paradigmatic polaritonic systems. We leverage the Psi4Numpy framework to yield open-source and accessible reference implementations of these methods.

\section{INTRODUCTION}

The interaction between molecular excitations and nanoconfined photons can produce the requisite strong interactions for polaritonic chemistry ${ }^{1-29}$. Motivated by a desire to provide a realistic picture of the molecular structure under the influence of strong photonic interaction, there has been a recent surge in activity focused on merging ab initio molecular electronic structure theory with cavity quantum electrodynamics (ab initio CQED) to provide an accurate and predictive model of polaritonic chemistry $9,24,27,30-35$. Such approaches can provide access to potential energy surfaces, couplings, and other properties of interest for simulating the structure and reactivity of polaritonoic chemical systems. Here we present a simple ab initio QED method for treating ground- and excited- polaritonic states with explicit inclusion of photonic lifetimes via a non-Hermitian cavity quantum electrodynamics - configuration interaction singles approach (NH-CQED-CIS). We implement this approach in the coherent state basis which results from solution of the CQED-Hartree-Fock (CQED-HF) equations. We endeavor to provide a detailed picture of the key equations and algorithmic considerations for both the CQED-HF and NH-CQED-CIS approach, and also provide reference implementations through the Psi4Numpy project ${ }^{36}$. We apply both methods to the analysis of polaritonic structure of several paradigmatic systems.

\section{THEORY}

We start with the Pauli-Fierz Hamiltonian in the dipole approximation in the length gauge, written in atomic units, following $24,27,31$ :

$$
\hat{H}=\hat{H}_{e}+\hat{H}_{p}+\hat{H}_{d s e}+\hat{H}_{e p},
$$

where

$$
\hat{H}_{e}=\sum_{i}^{N_{e}} \hat{T}_{e}\left(x_{i}\right)+\sum_{i}^{N_{e}} \sum_{A}^{N_{N}} \hat{V}_{e N}\left(x_{i} ; X_{A}\right)+\sum_{i}^{N_{e}} \sum_{j}^{N_{e}} \hat{V}_{e e}\left(x_{i}, x_{j}\right)+V_{N, N}
$$

with $\hat{T}_{e}\left(x_{i}\right)$ denoting the electronic kinetic energy operator for electron $i, \hat{V}_{e N}\left(x_{i} ; X_{A}\right)$ the (attractive) coulomb operator for electron $i$ and nucleus $A, \hat{V}_{e e}\left(x_{i}, x_{j}\right)$ the (repulsive) coulomb operator for electrons $i$ and $j$, and $V_{N, N}$ is the total (repulsive) coulomb potential between all of the nuclei. Within the BornOppenheimer approximation, $V_{N, N}$ is a constant, the nuclear kinetic energy is neglected, and the electron-nuclear attraction depends parametrically on the fixed nuclear coordinates. The photonic contribution is captured by the complex energy

$$
\hat{H}_{p}=\tilde{\omega} \hat{b}^{\dagger} \hat{b}
$$

and the photon-molecule intereaction contains a bilinear coupling term,

$$
\hat{H}_{e p}=-\sqrt{\frac{\tilde{\omega}}{2}}(\lambda \cdot(\hat{\mu}-\langle\mu\rangle))\left(\hat{b}^{\dagger}+\hat{b}\right),
$$

and a quadratic dipole self energy term,

$$
\hat{H}_{d s e}=\frac{1}{2}(\lambda \cdot(\hat{\mu}-\langle\mu\rangle))^{2} .
$$

In the above, $\hat{b}^{\dagger}$ and $\hat{b}$ are the bosonic raising/lowering operators for the photonic degrees of freedom, and $\tilde{\omega}=\omega-i \frac{\gamma}{2}$ is a complex frequency of the photon with the real part $\omega$ being related to the energy of the photon, and the imaginary part $\gamma$ being related to the dissipation rate of the photonic degree of freedom ${ }^{18,25,37}$. The term $\langle\mu\rangle$ represents the ground state molecular dipole expectation value which has cartesian components $\xi \in\{x, y, z\}$. A given $\xi$ component of the dipole operator has the form $\hat{\mu}^{\xi}=\sum_{i}^{N_{e}} \hat{\mu}^{\xi}\left(x_{i}\right)+\sum_{A}^{N_{N}} \mu_{n u c}^{\xi}\left(x_{A}\right)$, where $\hat{\mu}^{\xi}\left(x_{i}\right)$ is an operator that depends on electronic coordinates and within the Born-Oppenheimer approximation, we treat $\mu_{n u c}^{\xi}\left(x_{A}\right)$ as a function of the nuclear coordinates rather than a quantum mechanical operator. Note that the shift of the 
Hamiltonian by $\langle\mu\rangle$ results from the transformation to the coherent state basis ${ }^{24}$. To compute $\langle\mu\rangle^{\xi}$, we can use a single Slater determinant approximation to the ground electronic state,

$$
\begin{aligned}
\langle\mu\rangle^{\xi} & =\mid\left\langle\Phi_{0}\left|\sum_{i}^{N_{e}} \hat{\mu}^{\xi}\left(x_{i}\right)+\sum_{A}^{N_{N}} \mu_{n u c}^{\xi}\left(x_{A}\right)\right| \Phi_{0}\right\rangle \\
& =\left\langle\Phi_{0}\left|\sum_{i}^{N_{e}} \hat{\mu}^{\xi}\left(x_{i}\right)\right| \Phi_{0}\right\rangle+\left\langle\Phi_{0} \mid \Phi_{0}\right\rangle \sum_{A}^{N_{N}} \mu_{n u c}^{\xi}\left(x_{A}\right) \\
& =\sum_{i}^{N_{\text {occ }}} \mu_{i i}^{\xi}+\sum_{A}^{N_{N}} \mu_{n u c}^{\xi}\left(x_{A}\right),
\end{aligned}
$$

where $\mu_{i i}^{\xi}=\left\langle i\left|\hat{\mu}^{\xi}\right| i\right\rangle$ denotes the $\xi$-component of the occupied molecular dipole integrals and $\sum_{A}^{N_{N}} \mu_{n u c}^{\xi}\left(x_{A}\right)$ is the $\xi$ component of the nuclear dipole moment defined by the nuclear coordinates and atomic charges in the molecule. As we will see in the following section, solving the CQED-RHF equations will entail iterative updates to $\langle\mu\rangle$ with the CQEDRHF orbitals.

\section{A. CQED-RHF}

As our first step in solving for the energy eigenstates of Eq. 1, we follow Ref. 24 and 27 and introduce a product wavefunction between an electronic Slater determinant (which in practice may be initialized using a canonical RHF wavefunction) and a zero-photon number state,

$$
|R\rangle=\left|\Phi_{0}\right\rangle|0\rangle .
$$

To develop CQED-RHF theory, we examine the expectation value of Eq.1 with respect to Eq. 7,

$$
\begin{aligned}
& \left\langle R\left|\hat{H}_{e p}\right| R\right\rangle+\left\langle 0\left|\hat{H}_{p}\right| 0\right\rangle+\left\langle\Phi_{0}\left|\hat{H}_{e}+\hat{H}_{d s e}\right| \Phi_{0}\right\rangle \\
& =\left\langle\Phi_{0}\left|\hat{H}_{e}\right| \Phi_{0}\right\rangle+\left\langle\Phi_{0}\left|\hat{H}_{d s e}\right| \Phi_{0}\right\rangle,
\end{aligned}
$$

where we see that the terms involving $\hat{H}_{p}$ and $\hat{H}_{e p}$ vanish, and the expectation value of $\hat{H}_{e}$ is analogous to the ordinary RHF energy. To evaluate the expectation value of $\hat{H}_{d s e}$, we can first expand $\hat{H}_{d s e}$ in terms of the dipole operator (with electronic and nuclear contributions) and dipole expectation values as follows:

$$
\begin{aligned}
\hat{H}_{d s e} & =\sum_{\xi, \xi^{\prime}} \sum_{i, j>i} \lambda^{\xi} \lambda^{\xi^{\prime}} \hat{\mu}^{\xi}\left(x_{i}\right) \hat{\mu}^{\xi^{\prime}}\left(x_{j}\right) \\
& -\frac{1}{2} \sum_{\xi, \xi^{\prime}} \sum_{i} \lambda^{\xi} \lambda^{\xi^{\prime}} \hat{Q}^{\xi \xi^{\prime}}\left(x_{i}\right) \\
& +\left(\lambda \cdot \mu_{n u c}-\lambda \cdot\langle\mu\rangle\right) \sum_{\xi} \sum_{i} \lambda^{\xi} \hat{\mu}^{\xi}\left(x_{i}\right) \\
& +\frac{1}{2}\left(\lambda \cdot \mu_{n u c}\right)^{2}-(\lambda \cdot\langle\mu\rangle)\left(\lambda \cdot \mu_{n u c}\right)+\frac{1}{2}(\lambda \cdot\langle\mu\rangle)^{2}
\end{aligned}
$$

In the above expansion of $\hat{H}_{d s e}$ we have specifically indicated that the product of electronic dipole operators contains 2electron contributions when $i \neq j$, and 1-electron quadrupole contributions when $i=j$. The quadrupole contributions arise from the fact that $\hat{\mu}^{\xi}\left(x_{i}\right) \hat{\mu}^{\xi^{\prime}}\left(x_{i}\right)=-\hat{Q}^{\xi \xi^{\prime}}\left(x_{i}\right)$. Furthermore, a one-electron term arises that contains the electronic dipole operator scaled by $\lambda \cdot \mu_{n u c}-\lambda \cdot\langle\mu\rangle$, where again $\langle\mu\rangle$ will be iteratively updated during the QED-RHF procedure.

To solve the CQED-RHF equations, the additional oneelectron terms above will be added to $H_{\text {core }}$ and the additional two-electron terms above will be included in the densitymatrix dependent terms in the Fock operator:

$$
F_{\mu v}=H_{\mu v}+G_{\mu v}
$$

where

$$
\begin{aligned}
H_{\mu v} & =h_{\mu v}-\frac{1}{2} \sum_{\xi, \xi^{\prime}} \lambda^{\xi} \lambda^{\xi^{\prime}} Q_{\mu v}^{\xi \xi^{\prime}} \\
& +\left(\lambda \cdot \mu_{n u c}-\lambda \cdot\langle\mu\rangle\right) \sum_{\xi} \lambda^{\xi} \mu_{\mu v}^{\xi}
\end{aligned}
$$

and

$$
\begin{aligned}
G_{\mu v} & =(2(\mu v \mid \lambda \sigma)-(\mu \lambda \mid v \sigma)) D_{\lambda \sigma} \\
& +\left(\sum_{\xi \xi^{\prime}} \lambda^{\xi} \lambda^{\xi^{\prime}}\left(2 \mu_{\mu v}^{\xi} \mu_{\lambda \sigma}^{\xi^{\prime}}-\mu_{\mu \lambda}^{\xi} \mu_{v \sigma}^{\xi^{\prime}}\right)\right) D_{\lambda \sigma},
\end{aligned}
$$

leading to the total QED-RHF energy being

$$
E_{Q E D-R H F}=\left(F_{\mu v}+H_{\mu v}\right) D_{\mu v}+V_{N, N}+d_{c}
$$

where

$$
d_{c}=\frac{1}{2}\left(\lambda \cdot \mu_{n u c}\right)^{2}-(\lambda \cdot\langle\mu\rangle)\left(\lambda \cdot \mu_{n u c}\right)+\frac{1}{2}(\lambda \cdot\langle\mu\rangle)^{2} .
$$

For clarity, we briefly outline the CQED-RHF algorithm below:

1. Compute kinetic, nuclear attraction, electron repulsion, dipole, and quadrupole integrals in $\mathrm{AO}$ basis

\section{Perform canonical RHF calculation}

3. Initialize $\mathbf{D}$ and $\langle\mu\rangle$ from canonical RHF wavefunction

4. Augment core Hamiltonian with the dipole and quadrupole terms in Eq. 11

5. Augment the Fock matrix by contracting products of dipole integrals over current density matrix in Eq 12

6. Compute SCF energy through Eq. 15

7. Diagonalize Fock matrix and update density matrix

8. Check for convergence, if not converged, return to step 5. 


\section{B. Non-Hermitian QED-CIS in the CQED-RHF basis}

A mean-field description of the excited states of the molecular system strongly interacting with photonic degrees of freedom may be obtained through a configuration interaction singles (CIS) ansatz for the excited-states. Here we formulated a non-Hermitian version of such an ansatz, NH-CQED-CIS, that incorporates the dissipative features of the photonic degrees of freedom. In our presentation, we formulate $\mathrm{NH}$ CQED-CIS in the coherent state basis using the orbitals that result from the CQED-RHF approach outlined above.

The polaritonic energy eigenfunctions for state $I$ in the NH-CQED-CIS ansatz can be written as a linear combination of the CQED-RHF ground-state and products of all possible single excitations out of the CQED-RHF ground state. The CQED-RHF ground state involves the product of an electronic Slater determinant with the photon vacuum state, single excitations can occur as electronic excitations from an occupied orbital $i$ to a virtual orbital $a$, the raising of the photon number state from $|0\rangle \rightarrow|1\rangle$, or both. We therefore write the NHQED-CIS wavefunction for state $I$ as

$$
\Psi_{I}=c_{0}^{0}\left|\Phi_{0}\right\rangle|0\rangle+c_{0}^{1}\left|\Phi_{0}\right\rangle|1\rangle+\sum_{i, a} c_{i a}^{0}\left|\Phi_{i}^{a}\right\rangle|0\rangle+\sum_{i, a} c_{i a}^{1}\left|\Phi_{i}^{a}\right\rangle|1\rangle
$$

where the coefficients $c$ denote the contribution of a given term to the wavefunction, where we have denoted the electronic excitations in the subscript and the photonic excitations in the superscript of these coefficients. These coefficients, and the corresponding energy eigenvalues for a given NH-CQEDCIS state $I$ may be obtained by diagonalizing the Hamiltonian matrix built in the basis of the CQED-RHF and singly-excited states from Eq. 15. We use a spin-adapted basis of singly excited electronic states, such that $\left|\Phi_{i}^{a}\right\rangle=\frac{1}{\sqrt{2}}\left(\left|\Phi_{i \alpha}^{a \alpha}\right\rangle+\left|\Phi_{i \beta}^{a \beta}\right\rangle\right)$. There are three classes of matrix elements that contribute to the Hamiltonian matrix; we write these matrix elements after shifting the total Hamiltonian matrix in Eq. 1 by $E_{C Q E D-R H F}$. Matrix elements involving the CQED-RHF electronic Slater determinant $\left|\Phi_{0}\right\rangle$ and photonic states $|s\rangle$ and $|t\rangle$, where $s, t \in$ $\{0,1\}$ involve only the (complex) photonic energy,

$$
\left\langle s\left|\left\langle\Phi_{0}\left|\hat{H}-E_{C Q E D-R H F}\right| \Phi_{0}\right\rangle\right| t\right\rangle=\tilde{\omega} t \delta_{s t} .
$$

Matrix elements coupling $\left|\Phi_{0}\right\rangle|s\rangle$ to $\left|\Phi_{i}^{a}\right\rangle|t\rangle$ involve only the $\hat{H}_{e p}$ contributions:

$$
\begin{aligned}
& \left\langle s\left|\left\langle\Phi_{0}\left|\hat{H}-E_{C Q E D-R H F}\right| \Phi_{i}^{a}\right\rangle\right| t\right\rangle= \\
& -\sqrt{\tilde{\omega}} \sqrt{t+1} \delta_{s, t+1} \sum_{\xi} \lambda \xi \mu_{i a}^{\xi} \\
& --\sqrt{\tilde{\omega}} \sqrt{t} \delta_{s, t-1} \sum_{\xi} \lambda \xi \mu_{i a}^{\xi} .
\end{aligned}
$$

Matrix elements coupling different singly excited electronic and/or photonic states involve all terms of the Hamiltonian, including the usual CIS terms:

$$
\begin{aligned}
& \left\langle s\left\langle\Phi_{i}^{a}\left|\hat{H}-E_{C Q E D-R H F}\right| \Phi_{j}^{b}\right\rangle \mid t\right\rangle \\
& =\left(\varepsilon_{a}-\varepsilon_{i}+d_{c}+\tilde{\omega} t\right) \delta_{i j} \delta_{a b} \delta_{s t} \\
& +\delta_{s t}(2(i a \mid j b)-(i j \mid a b)) \\
& +2 \delta_{s t} \sum_{\xi, \xi^{\prime}} \lambda^{\xi} \lambda^{\xi^{\prime}} \mu_{i a}^{\xi} \mu_{j b}^{\xi} \\
& -\delta_{s t} \sum_{\xi, \xi^{\prime}} \lambda^{\xi} \lambda^{\xi^{\prime}} \mu_{i j}^{\xi} \mu_{a b}^{\xi} \\
& +\sqrt{t+1} \delta_{s, t+1} \delta_{i j} \delta_{a b} \sqrt{\frac{\tilde{\omega}}{2}} \lambda \cdot\langle\mu\rangle \\
& +\sqrt{t} \delta_{s, t-1} \delta_{i j} \delta_{a b} \sqrt{\frac{\tilde{\omega}}{2}} \lambda \cdot\langle\mu\rangle \\
& -\sqrt{t+1} \delta_{s, t+1} \delta_{i j} \delta_{a b} \sqrt{\frac{\tilde{\omega}}{2}} \sum_{\xi} \sum_{k} \lambda \xi \mu_{k k}^{\xi} \\
& -\sqrt{t} \delta_{s, t-1} \delta_{i j} \delta_{a b} \sqrt{\frac{\tilde{\omega}}{2}} \sum_{\xi} \sum_{k} \lambda^{\xi} \mu_{k k}^{\xi} \\
& -\sqrt{t+1} \delta_{s, t+1} \delta_{i j} \sqrt{\frac{\tilde{\omega}}{2}} \sum_{\xi} \lambda^{\xi} \mu_{a b}^{\xi} \\
& -\sqrt{t} \delta_{s, t-1} \delta_{i j} \sqrt{\frac{\tilde{\omega}}{2}} \sum_{\xi} \lambda^{\xi} \mu_{a b}^{\xi} \\
& +\sqrt{t+1} \delta_{s, t+1} \delta_{a b} \sqrt{\frac{\tilde{\omega}}{2}} \sum_{\xi} \lambda^{\xi} \mu_{i j}^{\xi} \\
& +\sqrt{t} \delta_{s, t-1} \delta_{a b} \sqrt{\frac{\tilde{\omega}}{2}} \sum_{\xi} \lambda^{\xi} \mu_{i j}^{\xi}
\end{aligned}
$$

\section{REFERENCE IMPLEMENTATIONS}

We provide reference implementations using Psi4Numpy ${ }^{36}$, which provides a simple NumPy interface to the Psi $4^{38}$ quantum chemistry engine. The code for these reference implementations can be freely accessed in the hilbert package ${ }^{39,40}$. Furthermore, to provide a no-installation option for interested users to experiment with these implementations, we utilize the ChemCompute project $^{41}$ to host the illustrative calculations discussed in the Results section below. Interested users can navigate to https://chemcompute.org/register to register for a free ChemCompute account. Following registration, interested users can run calculations described in Figure 1 and Table I in the results section using the link in Ref. 42 , and can run calculations described in Figure 2 and 3 using the link within Ref. 43.

\section{RESULTS}

We apply the CQED-RHF and NH-CQED-CIS approaches to a few simple paradigmatic polaritonic chemical systems. 
First we examine the ground-state of formaldehyde strongly coupled to a single photon mode, which has been explored in by several groups that have been developing density functional theory-based $a b$ initio-QED methods ${ }^{9,35}$. We optimize the geometry of lone formaldehyde at the RHF/cc-pVDZ level and perform all calculations at that geometry. At this level, the RHF ground-state has a dipole moment oriented purely along the $z$-axis with $\langle\mu\rangle_{z}=-1.009$ atomic units. We examine the impacts of photon polarization along both the $y-$, $z-$ (or both) axes in Figure 1. In particular, we scan values of the magnitude of $|\lambda|$ in the range $[0,0.2]$ atomic units in increments of 0.02 atomic units for three different polarizations: $\lambda_{y}=(0,|\lambda|, 0), \lambda_{z}=(0,0,|\lambda|)$, and $\lambda_{y z}=\left(0, \frac{|\lambda|}{\sqrt{2}}, \frac{|\lambda|}{\sqrt{2}}\right)$. We note that the electric field vector enters explicitly into the CQED-RHF equations while the photon frequency does not, so we do not report a specific value of $\tilde{\omega}$ for these calculations.

We see, not surprisingly, that the CQED-RHF energy increases monotonically with the magnitude $\lambda_{z}$, and interestingly, shows the same qualitative behavior (albeit slightly less dramatically) with increasing magnitude of $\lambda_{y}$ (see top panel of Figure 1). We first decompose the total CQED-RHF energy into the canonical RHF contribution,

$$
E_{R H F}=\left(2 h_{\mu v}+2(\mu \nu \mid \lambda \sigma) D_{\lambda \sigma}-(\mu \lambda \mid v \sigma) D_{\lambda \sigma}\right) D_{\mu \nu}
$$

and the Pauli-Fierz contribution that includes the remaining terms in the CQED-RHF Fock operator traced against the converged CQED-RHF density matrix (with elements $D_{\mu \nu}$ denoted above).

Not surprisingly, we see that the Pauli-Fierz contribution shows the most dramatic scaling with the electric field magnitude, comprising the majority of the energy change, but we also see that the canonical RHF contribution is modifed by the electric field, as well. We present a more granular decomposition of these energetic contributions only for the largest field magnitude $|\lambda|=0.2$ atomic units in Table I.

\begin{tabular}{c|c|c|c|c|c|c}
$\Delta E$ (a.u.) & $\% \Delta_{1 E}\left|\% \Delta_{2 E}\right| \% \Delta_{1 d e}\left|\% \Delta_{1 q e}\right| \% \Delta_{2 d e} \mid \% \Delta_{d_{c}}$ \\
\hline \multicolumn{7}{c}{$\lambda_{y}$} \\
\hline 0.135 & -210 & 213 & 0 & 210 & -113 & 0 \\
\hline 0.161 & -169 & 172 & -23 & 440 & -331 & 11 \\
\hline \multicolumn{7}{c}{$\lambda_{y z}$} \\
\hline 0.148 & -185 & 188 & -12 & 333 & -230 & 6 \\
\hline
\end{tabular}

TABLE I. Change in total CQED-RHF energy ( $\Delta E$ in atomic units) and $\%$ relative changes in different contributions to the total CQEDRHF energy for three different polarizations of a photonic mode with magnitude $|\lambda|=0.2$ atomic units.

From Eq. 7, we see that only the photon vacuum contributes to the CQED-RHF wavefunction. However, in Eq. (17), we see that the CQED-RHF wavefunction can couple to states which involve singly-excited electronic configurations and singly-occupied photon states. This coupling can lower the energy of the lowest energy eigenstate of the CQED-CIS Hamiltonian relative to the ground-state determined by the CQED-RHF method.

We illustrate this point through the ground-state potential
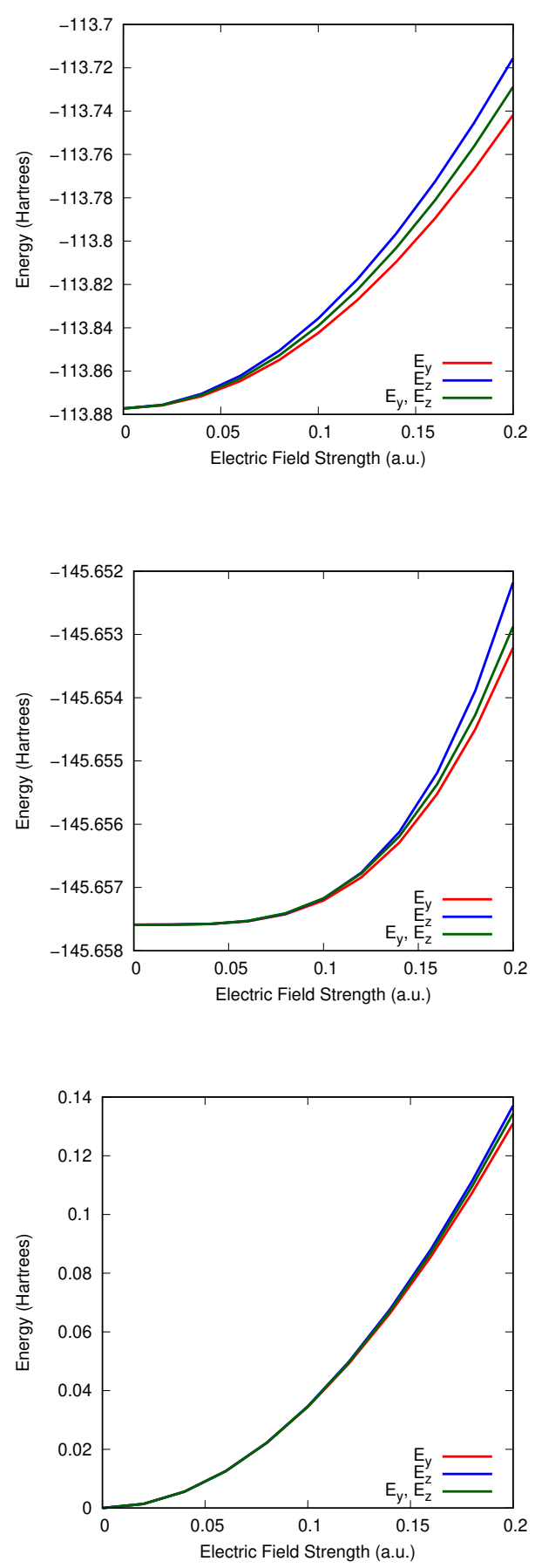

FIG. 1. (Top) Total CQED-RHF energy, (middle) canonical RHF contribution to the total energy, (bottom) Pauli-Fierz contribution to the total energy as a function of $|\lambda|$ along the $y$ - and/or $z$ - axes.

energy surface of the $\mathrm{MgH}^{+}$diatomic cation coupled to a photon that is on resonance with the ground-state singlet $(|X\rangle)$ to first excited-state singlet $(|A\rangle)$ when the $\mathrm{MgH}^{+}$bondlength is approximately 2.2 Angstroms $^{23}$. We consider the molecule to be oriented along with $z$ axis, which is parallel to the polarization vector of the photon that has magnitude $\lambda_{z}=0.075$ 


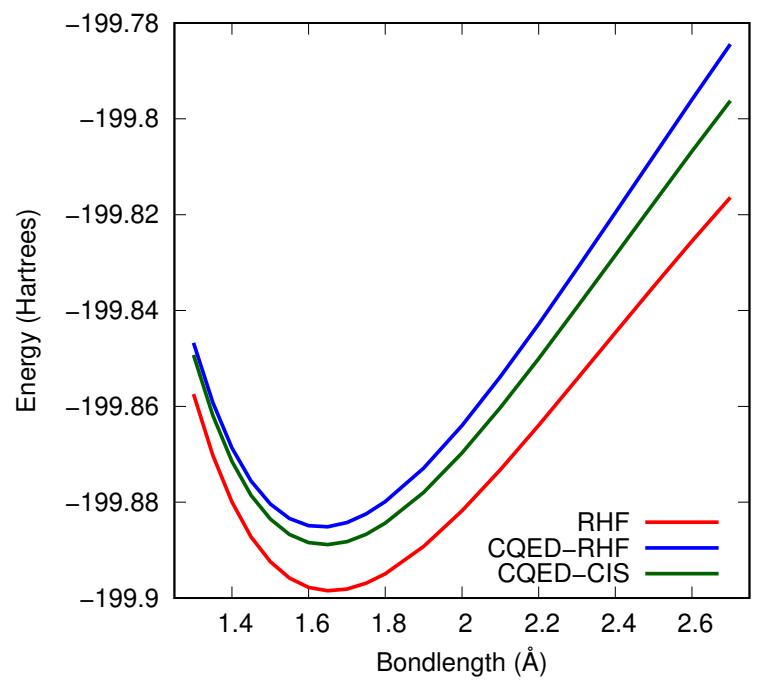

FIG. 2. Ground-state potential energy surface of $\mathrm{MgH}+$ coupled to a photon resonant with $X \rightarrow A$ transition. We see that the photonic coupling raises the energy relative to the lone molecular system (as computed at the RHF/cc-pVDZ level). Coupling between the CQED-RHF wavefunction and singly-excited electronic and photonic configurations stabilizes the CQED-CIS ground-state relative to the CQED-RHF ground-state.

atomic units. We compute the potential energy surface of this system at the RHF/cc-pVDZ, CQED-RHF/cc-pVDZ, and CQED-CIS/cc-pVDZ level and plot the results in Figure 2.

As a final illustrative example, we consider the upperpolariton $(|U P\rangle)$ and lower-polariton $(|L P\rangle)$ states that emerge from coupling $\mathrm{MgH}^{+}$to a photon resonant with the $|X\rangle \rightarrow|A\rangle$ transition. We again consider a $z$-polarized photon with magnitude $\lambda_{z}=0.0125$ atomic units. This time, we allow the photon to have a complex energy where the imaginary part accounting for the finite lifetime can also be related to the energy uncertainty of the photon. We consider the real part of the photon energy to be $4.75 \mathrm{eV}$, and we consider the imaginary part to be either $0 \mathrm{eV}$ or $0.22 \mathrm{eV}$ as shown in the top and bottom panels of Figure 3, respectively. In addition to computing these polariton surfaces at the CQED-CIS/cc-pVDZ level, we also fit a 3-level model Pauli-Fierz Hamiltonian from ordinary CIS/cc-pVDZ potential energy surfaces:

$$
H=\left(\begin{array}{ccc}
E_{X}+\frac{\left(\lambda \cdot\left\langle\mu_{X}\right\rangle\right)^{2}}{2} & 0 & 0 \\
0 & E_{X}+\tilde{\omega}+\frac{\left(\lambda \cdot\left\langle\mu_{X}\right\rangle\right)^{2}}{2} & \sqrt{\frac{\tilde{\omega}}{2}} \lambda \cdot \mu_{X A} \\
0 & \sqrt{\frac{\tilde{\omega}}{2}} \lambda \cdot \mu_{X A} & E_{A}+\frac{\left(\lambda \cdot\left\langle\mu_{A}\right\rangle\right)^{2}}{2} .
\end{array}\right)
$$

The polaritonic surfaces obtained from diagonalizing Eq. (20) are referred to as the 'Model LP' and 'Model UP' surfaces in Figure 3. We see with a pure real photon energy, the $|L P\rangle$ and $|U P\rangle$ surfaces experience a strong splitting in the region where the $|X, 1\rangle$ state (the ground-state plus a photon) crosses the $|A, 0\rangle$ state (the first excited-state without a photon). However,
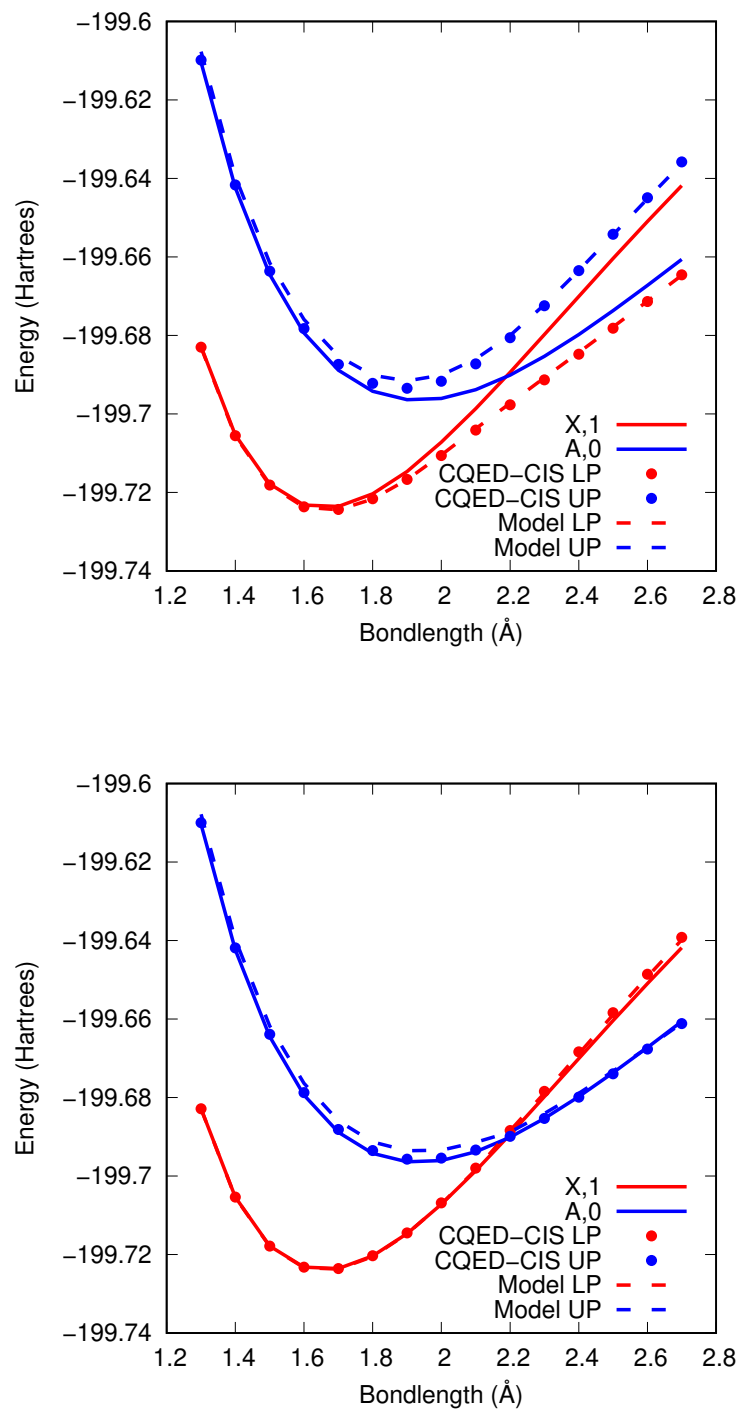

FIG. 3. Polaritonic surface of $\mathrm{MgH}+$ coupled to a photon with (top) $\hbar \tilde{\omega}=4.75 \mathrm{eV}$ and (bottom) $\hbar \tilde{\omega}=4.75-0.22 \mathrm{eV}$ with $\lambda_{z}=0.0125$ atomic units. We see evidence of strong coupling via splitting of the surfaces where the $|X, 1\rangle$ and $|A, 0\rangle$ states are resonant, when the photon energy is pure real, and we see the splitting vanish when the imaginary part of the photon energy is large compared to the interaction energy.

for the strongly dissipative photon, we see that both the model and CQED-CIS curves closely approximate the CIS curves for the lone molecules, which signals that this system is not in the strong-coupling regime because of the lossiness associated with the photon ${ }^{25}$. 


\section{CONCLUSIONS}

We combined $a b$ initio molecular electronic Hamiltonians with a cavity quantum electrodynamics (CQED) model for dissipative photonic modes and apply mean-field theories to the ground- and excited-states of resulting polaritonic systems. In particular, we developed a restricted HartreeFock theory for the mean-field ground-state and a nonHermitian configuration interaction singles theory for meanfield excited-states of the molecular system strongly interacting with a photonic mode, and applied these methods to several paradigmatic polaritonic systems. We leveraged the Psi4Numpy ${ }^{36}$ framework to yield open-source and accessible reference implementations of these methods.

\section{ACKNOWLEDGMENTS}

JM and JJF Acknowledges support from the Research Corporporation for Scientific Advancement Cottrell Scholar Award and the NSF CAREER Award CHE-2043215. Computational resources were provided in part by the MERCURY consortium (http://mercuryconsortium.org/) under NSF grants CHE-1229354, CHE-1662030, and CHE-2018427. We gratefully acknowledge A. E. DePrince III for numerous helpful discussions and access to benchmark results for the CQEDRHF method.

${ }^{1}$ J. A. Hutchison, T. Schwartz, C. Genet, E. Devaux, and T. W. Ebbesen, "Modifying chemical landscapes by coupling to vacuum fields," Angew. Chem. Int. Ed. Engl. 13, 1592-1596 (2012).

${ }^{2}$ J. Galego, F. J. Garcia-Vidal, and J. Feist, "Cavity-induced modifications of molecular structure in the strong-coupling regime," Phys. Rev. X 5, 041022 (2015).

${ }^{3}$ T. W. Ebbesen, "Hybrid light-matter states in a molecular and material science perspective," Acc. Chem. Res. 49, 2403-2412 (2016).

${ }^{4}$ F. Herrera and F. C. Spano, "Cavity-controlled chemistry in molecular ensembles,” Phys. Rev. Lett. 116, 238301 (2016).

${ }^{5}$ N. M. Hoffmann, H. Appel, A. Rubio, and N. T. Maitra, "Light-matter interactions via the exact factorization approach," Eur. Phys. J. B 91, 180 (2018).

${ }^{6}$ J. Feist, J. Galego, and F. J. Garcia-Vidal, "Polaritonic chemistry with organic molecules," ACS Photonics 5, 205-216 (2018).

${ }^{7}$ L. A. Martinez-Martinez, R. F. Ribeiro, J. Campos-Gonzalez-Angulo, and J. Yuen-Zhou, "Can ultrastrong coupling change ground-state chemical reactions?" ACS Photonics 5, 167-176 (2018).

${ }^{8}$ B. Munkhbat, M. Wersäll, D. G. Baranov, T. J. Antosiewicz, and T. Shegai, "Suppression of photo-oxidation of organic chromophores by strong coupling to plasmonic nanoantennas," Sci. Adv. 4, eaas9552 (2018).

${ }^{9}$ J. Flick and P. Narang, "Cavity-correlated electron-nuclear dynamics from first principles,” Phys. Rev. Lett. 121, 113002 (2018).

${ }^{10}$ J. Fregoni, G. Granucci, E. Coccia, M. Persico, and S. Corni, "Manipulating azobenzene photoisomerization through strong light-molecule coupling," Nat. Commun. 9, 4688 (2018).

${ }^{11}$ L. A. Martínez-Martínez, M. Du, R. F. Ribeiro, S. Kéna-Cohen, and J. Yuen-Zhou, "Polariton-assisted singlet fission in acene aggregates," J. Phys. Chem. Lett. 9, 19511957 (2018).

${ }^{12}$ L. A. Martínez-Martínez, E. Eizner, S. Kéna-Cohen, and J. Yuen-Zhou, "Triplet harvesting in the polaritonic regime: A variational polaron approach,” J. Chem. Phys. 151, 054106 (2019).

${ }^{13}$ O. D. Stefano, A. Settineri, V. Macri, L. Garziano, R. Stassi, S. Savasta, and F. Nori, "Resolution of gauge ambiguities in ultrastrong-coupling cavity quantum electrodynamics," Nat. Phys. 15, 803-808 (2019).

${ }^{14}$ M. Du, R. F. Ribeiro, and J. Yuen-Zhou, "Remote control of chemistry in optical devices," Chem. Cell. Press 5, 1167-1181 (2019).
${ }^{15}$ A. Mandal and P. Huo, "Investigating new reactivities enabled by polariton photochemistry," J. Phys. Chem. Lett. 10, 5519-5529 (2019).

${ }^{16}$ A. Mandal, T. D. Krauss, and P. Huo, "Polariton-mediated electron transfer via cavity quantum electrodynamics," J. Phys. Chem. B (2020).

${ }^{17}$ M. A. D. Taylor, A. Mandal, W. Zhou, and P. Huo, "Resolution of gauge ambiguities in molecular cavity quantum electrodynamics," Phys. Rev. Lett. (2020).

${ }^{18}$ S. Felicetti, J. Fregoni, T. Schnappinger, S. Reiter, R. de Vivie-Riedle, and J. Feist, "Photoprotecting uracil by coupling with lossy nanocavities," J. Phys. Chem. Lett. 11, 8810 (2020).

${ }^{19}$ J. Fregoni, S. Corni, M. Persico, and G. Granucci, "Photochemistry in the strong coupling regime: A trajectory surface hopping scheme," J. Comput. Chem. 41, 2033-2044 (2020).

${ }^{20}$ J. Fregoni, G. Granucci, M. Persico, and S. Corni, "Strong coupling with light enhances the photoisomerization quantum yield of azobenzene," Chem 6, 250-265 (2020).

${ }^{21}$ I. S. Ulusoy and O. Vendrell, "Dynamics and spectroscopy of molecular ensembles in a lossy microcavity," J. Chem. Phys. 153, 044108 (2020).

${ }^{22}$ N. M. Hoffman, L. Lacombe, A. Rubio, and N. T. Maitra, "Effect of many modes on self-polaritzation and photochemical suppression in cavities," $\mathrm{J}$. Chem. Phys. 153, 104103 (2020).

${ }^{23}$ E. Davidsson and M. Kowalewski, "Simulating photodissociation reactions in bad cavities with the lindblad equation," J. Chem. Phys. 153, 234304 (2020).

${ }^{24}$ T. S. Haugland, E. Ronca, E. F. Kjønstad, A. Rubio, and H. Koch, "Coupled cluster theory for molecular polaritons: Changing ground and excited states," Phys. Rev. X 10, 041043 (2020).

${ }^{25}$ P. Antoniou, F. Suchanek, J. F. Varner, and J. J. F. IV, "Role of cavity losses on nonadiabatic couplings and dynamics in polaritonic chemistry," J. Phys. Chem. Lett. 11, 9063-9069 (2020).

${ }^{26}$ M. H. Farag, A. Mandal, and P. Huo, "Polariton induced conical intersection and berry phase," Phys. Chem. Chem. Phys. 23, 16868 (2021).

${ }^{27}$ A. E. D. III, "Cavity-modulated ionization potentials and electron affinities from quantum electrodynamics coupled-cluster theory," J. Chem. Phys. 154, 094112 (2021).

${ }^{28}$ M. Du, J. C. G. Angulo, and J. Yuen-Zhou, "Nonequilibrium effects of cavity leakage and vibrational dissipation in thermally-activated polariton chemistry," J. Chem. Phys. 154, 084108 (2021).

${ }^{29}$ J. Torres-Sánchez and J. Feist, "Molecular photodissociation enabled by ultrafast plasmon decay,” J. Chem. Phys. 154, 014303 (2021).

${ }^{30}$ I. V. Tokatly, "Time-dependent density functional theory for many-electron systems interacting with cavity photons," Phys. Rev. Lett. 110, 23301 (2013).

${ }^{31}$ M. Ruggenthaler, N. Tancogne-Dejean, J. Flick, H. Appel, and A. Rubio, "From a quantum-electrodynamical light-matter description to novel spectroscopies," Nat. Rev. Chem. 2, 0118 (2018).

${ }^{32}$ J. Flick and P. Narang, "Ab initio polaritonic potential-energy surfaces for excited-state nanophotonics and polaritonic chemistry,' J. Chem. Phys. 153, 094116 (2020).

${ }^{33}$ U. Mordovina, C. Bungey, K. Appel, P. J. Knowles, A. Rubio, and F. R. Manby, "Polaritonic coupled-cluster theory," Phys. Rev. Research 2, 023262 (2020).

${ }^{34}$ F. Buchholz, I. Theophilou, S. E. B. Nielsen, M. Ruggenthaler, and A. Rubio, "Reduced density-matrix approach to strong matter-photon interaction," ACS Photonics 6, 2694-2711 (2019).

${ }^{35}$ J. Yang, Q. Ou, Z. Pei, H. Wang, B. Weng, K. Mullen, and Y. Shao, "Quantum-electrodynamical time-dependent density functional theory. i. a gaussian atomic basis implementation," (2021), arXiv:2105.10530 [condmat.mtrl-sci].

${ }^{36}$ D. G. A. Smith, L. A. Burns, D. A. Sirianni, D. R. Nascimento, A. Kumar, A. M. James, J. B. Schriber, T. Zhang, B. Zhang, A. S. Abbott, E. J. Berquist, M. H. Lechner, L. A. Cunha, A. G. Heide, J. M. Waldrop, T. Y. Takeshita, A. Alenaizan, D. Neuhauser, R. A. King, A. C. Simmonett, J. M. Turney, H. F. Schaefer, F. A. Evangelista, A. E. D. III, T. D. Crawford, K. Patkowski, and C. D. Sherrill, "Psi4numpy: An interactive quantum chemistry programming environment for reference implementations and rapid development," J. Chem. Theor. Comput. 14, 3504-3511 (2018).

${ }^{37}$ C. L. Cortes, M. Otten, and S. K. Gray, "Non-hermitian approach for quantum plasmonics," J. Chem. Phys. 152, 084105 (2020). 
${ }^{38}$ J. M. Turney, A. C. Simmonett, R. M. Parrish, E. G. Hohenstein, F. Evangelista, J. T. Fermann, B. J. Mintz, L. A. Burns, J. J. Wilke, M. L. Abrams, N. J. Russ, M. L. Leininger, C. L. Janssen, E. T. Seidl, W. D. Allen, H. F. Schaefer, R. A. King, E. F. Valeev, C. D. Sherrill, and T. D. Crawford, "Psi4: An open-source ab initio electronic structure program," WIREs Comput. Mol. Sci. 2, 556 (2012).

${ }^{39}$ A. E. D. III, https://github. com/edeprince3/hilbert (2021), [Online; accessed 18-August-2021].
${ }^{40}$ J. McTague and J. J. F. IV, https://github.com/edeprince3/ hilbert/tree/master/src/psi4numpy (2021), [Online; accessed 18August-2021].

${ }^{41}$ M. J. Perri and S. H. Weber, "Web-based job submission interface for the gamess computational chemistry program,” J. Chem. Educ. 91, 2206-2208 (2014).

${ }^{42}$ J. McTague and J. J. F. IV, https://bit.ly/3iXFxvk (2021), [Online; accessed 18-August-2021].

${ }^{43}$ J. McTague and J. J. F. IV, https://bit.1y/3g9CIWr (2021), [Online; accessed 18-August-2021]. 